\title{
Simulation Modeling of Coarse Tracking System in Composite Axle System Based on Measured Operating Data
}

\author{
Congcong Liu", a, Shengli $\mathrm{Qu}^{2, \mathrm{~b}}$ and Haibing Wang ${ }^{3, \mathrm{c}}$ \\ ${ }^{1,2,3}$ Xidian University, No.2, South Taibai Road Xi'an City, Shaanxi Province,China

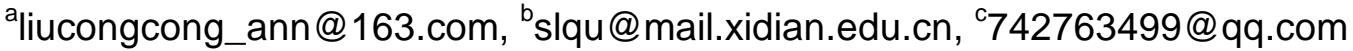

\begin{abstract}
Keywords: Modeling, Simulation, Simulation modeling, Composite axis system, Measured operating data
\end{abstract}

Abstract. The coarse turntable has been determined. An important prerequisite for designing the composite axis control system and selecting the device for the precision tracking system is to establish an accurate coarse tracking model. Only have a set of measured operating data with non-differentiable nonlinear links and unknown interference. The system identification is difficult to identify the nonlinear part and interference affect the identification accuracy. We propose a method based on the measured operating data, using the method of combining analysis and simulation modeling. The model structure of the system is determined by analyzing, the specific parameters, nonlinear and interference of the model are determined by simulation modeling. The obtained model is matched well with the measured data, and a more accurate nonlinear model is obtained.

\section{Introduction}

Composite axis tracking technique is one of the key technologies of laser weapon precision tracking and pointing [1]. It is very important to master this key technology. As shown in Fig.1, composite axis control system consists of two nested control systems [2], coarse tracking system nested precision tracking system, The FSM (Fast Steering Mirror) is usually inserted on a large inertia frame. Frame and coarse tracking components constitute a coarse tracking system, which mainly realizes the optical signal acquisition and tracking. The precision tracking system mainly corrects the residual error of the coarse tracking to ensure fast and high precision tracking [1].

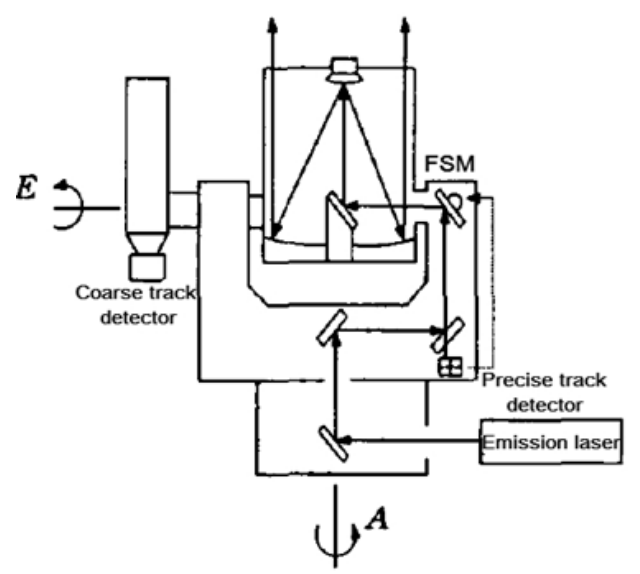

Fig. 1 Composite axis tracking system structure diagram

We only have a set of coarse tracking system measured operating data, the coarse tracking system has been in existence, the goal is to design the composite axis control system and to select the devices for the precision tracking system, an important premise of this work is to make a high-quality modeling for the coarse tracking system. After careful analysis, the coarse tracking system contains non-differentiable nonlinear links and unknown interference, which makes the modeling very difficult. System identification is simple and easy to implement for linear systems [3,4,5], but it is difficult for nonlinear systems, and the nonlinear part of the measured data enters the linear part, which will cause 
the linear model to be inaccurate $[6,7,8,9]$. Theoretical analysis modeling generally works well for linear systems and is not available for nonlinear systems [10,11,12].

Therefore, we propose a method based on the measured operating data, the analysis and simulation modeling combined to get a more accurate system model. That is, the model structure of the system is determined by analyzing, the specific parameters, nonlinear and interference of the model are determined by simulation modeling, and the process is progressive. In this paper, the method is applied to analyze and simulate the coarse tracking system of composite axis control system.

\section{Model structure Analysis}

The purpose of data analysis is to obtain the possible composition of the system. According to the measured data of the coarse turntable, we plot the measured input and output figure and the measured error figure, as respectively shown in Fig. 2 and Fig. 3.

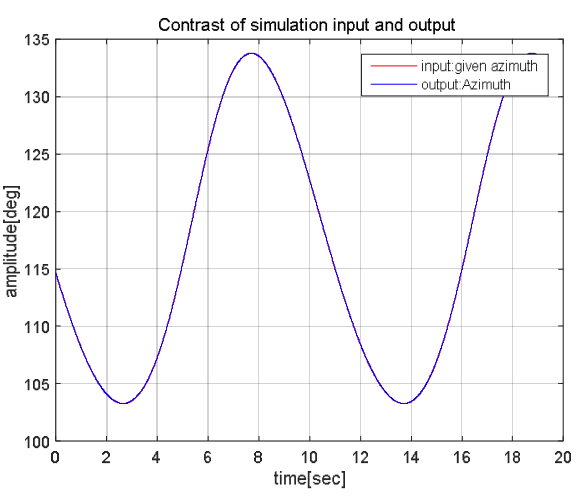

Fig. 2 Measured input and output



Fig. 3 Measured error for rough tracking system

As shown in Fig. 2, the measured input and output of the coarse turntable is a set of data that is not sufficiently excited, which makes identification difficult. It is obtained by using the coarse turntable with a photoelectric detector to track the photoelectric signal.

As shown in Fig. 3, the measured error with some regularity large jumps, we can determine the jump is a nonlinear link. The analysis shows that jump is a backlash. In addition, there is more jitter in Fig. 3 and we infer that there is interference in the input signal or in the coarse tracking system loop.

Linear links in each system is essential and the common tracking system is generally second-order or third-order system. Generally the second-order dominant pole can be used instead. Therefore, this paper based on the standard second-order system, according to the actual situation in this basic model to be amended to meet the design requirements.

Therefore, we analyzed that the model of the coarse tracking system may be composed of: standard second-order system, nonlinear backlash and interference.

\section{Simulation Modeling Process}

Here, we based on the measured operating data of the coarse tracking system, combining the possible composition of the model with the simulation modeling, and step by step to obtain a more accurate nonlinear model.

As the above analysis, the basic model of coarse tracking system is the standard second-order system, the closed-loop transfer function is:

$$
G(s)=\frac{\omega_{1}{ }^{2}}{s^{2}+2 \zeta \omega_{1} s+\omega_{1}^{2}}
$$

According to the input data of the measured operating data, the maximum rate of the input signal is $10.2716^{\circ} / \mathrm{s}$, the maximum tracking error is $0.011477^{\circ}=41.3172^{\prime \prime}$. 
Using the measured data to calculate the equivalent bandwidth of the coarse tracking is $f=220.691 \mathrm{~Hz}$. It should be emphasized that the actual turntable of bandwidth is very low, about $7-10 \mathrm{~Hz}$. It appears that the system contains a speed feedforward link, because the feedforward link has the ability to improve the system response speed. From the equivalent bandwidth can also be derived $\omega_{n}=1276.6$, the coarse tracking closed-loop transfer function Eq. 1 can be replaced by Eq. 2:

$$
G(s)=\frac{1276.6^{2}}{s^{2}+2 \times 0.69 \times 1276.6 s+1276.6^{2}} .
$$

Then, the open-loop transfer function is:

$$
G(s)=\frac{1276.6^{2}}{s^{2}+2 \times 0.69 \times 1276.6 s} .
$$

Next, use the simulation method to verify the above calculation is correct. The simulation block diagram of the coarse tracking is shown in Fig. 4. The Transfer Fen in the block diagram is Eq. 3; data is the input time sequence of the measured data; $k 1=\pi / 180$ and $k 2=180 \times 3600 / \pi$ are unit conversion factor. Due to limited space, the following directly gives the complete Simulink simulation diagram, as shown in Fig. 12.

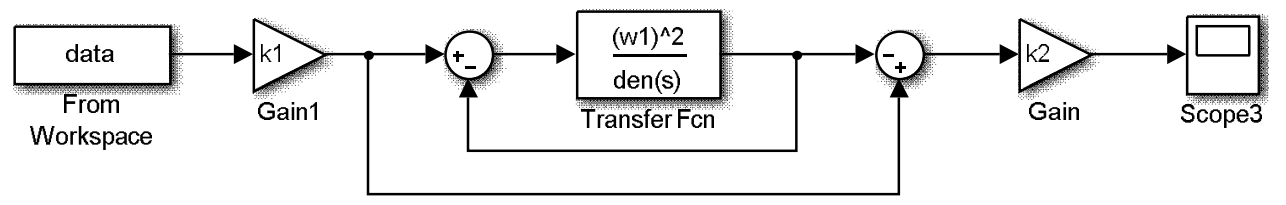

Fig. 4 Block diagram of coarse tracking

The simulation error of the coarse tracking system is shown in Fig. 5:

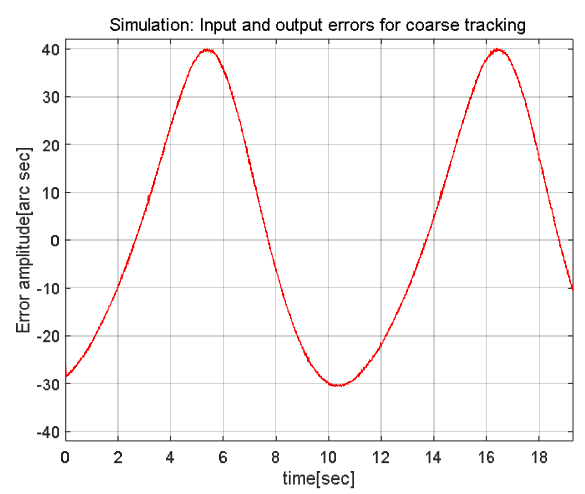

Fig. 5 Coarse tracking simulation error

As shown in Fig. 5, the error range is within 41" , in accordance with the design requirements.

From the analysis, the system is a second-order non-static error system with feedforward. Therefore, there must be a zero point in the transfer function. In this case, the closed-loop transfer function is:

$$
G(s)=\frac{\omega_{1}^{2}(1+k s)}{s^{2}+2 \zeta \omega_{1} s+\omega_{1}^{2}} .
$$

Next, adjusting the value of $k$ in Eq. 4 and making coarse tracking simulation error is less than $41^{\prime \prime}$. Where, $\omega_{1}=57.8455$ is the value corresponding to the coarse tracking system bandwidth of $10 \mathrm{~Hz}$ 
calculated; to take different values of $k$, to observe the coarse tracking system simulation error changes, as shown in Fig. 6.



Fig. 6 The variation of simulation error under different $\mathrm{k}$ values

The comparison between Fig. 6 and Fig. 3 shows that when $k=1.45 / 578.8455=0.0251$ is the most close to the measured error sequence of the coarse tracking system. The closed-loop transfer function of the coarse tracking system is:

$$
G(s)=\frac{57.8455^{2}(1+0.0251 s)}{s^{2}+2 \times 0.69 \times 57.8455 s+57.8455^{2}} .
$$

Following the above analysis, we determine the coarse tracking model for the second-order non-static error system with backlash to observe the coarse tracking simulation error, take the backlash size is $41^{\prime \prime}$. The backlash is added to the simulation, the simulation error shown in Fig. 7. There is a similar to the coarse tracking system measured error of the jump, closer to the measured data.



Fig. 7 Backlash is added to the simulation error

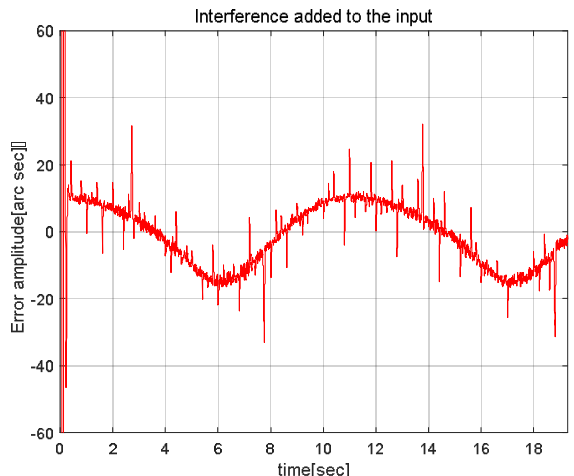

Fig. 8 Interference is added to the input

Now, compare Fig. 7 with Fig. 3, we need to add interference to the current model. We cannot determine the location of the interference. Therefore, in the input and loop add to the interference respectively, to explore whether they are the cause for error jitter.

(1). Interference in the input

In the simulation, we add the interference to the input. The simulation error of the coarse tracking is shown in Fig. 8. Next, compare Fig. 8with Fig. 3, and apparently see that the interference added to the input is far from the actual error. Here does not consider the input interference effects.

(2). Interference in the loop

In the simulation, we add the interference to the closed-loop. The simulation results are shown in Fig. 9. The interference is determined by continuous debugging as: 
Interference 1: non-unit random number, the maximum value of 10 seconds, the minimum -7 seconds, sampling time 0.2 seconds;

Interference 2: a sine wave with a frequency of $2 \mathrm{~Hz}$ and a gain of 0.00003 .

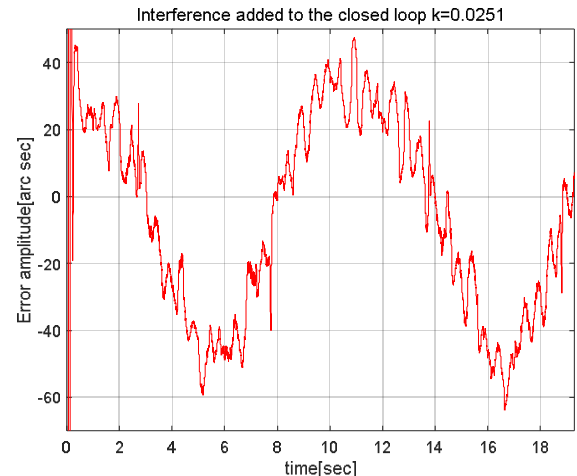

Fig. 9 Interference in the loop $k=0.0251$



Fig. 10 Interference in the loop $k=0.0242$

As shown in Fig. 9, jitter pattern is very similar to that guess; but the error is slightly larger and need to readjust the $k$ value in the Eq. 4 . By constantly adjusting, when $k=0.0242$, the error is close to the measured error, and the simulation error shown in Fig. 10. The simulation error and the measured error are compared, as shown in Fig. 11.

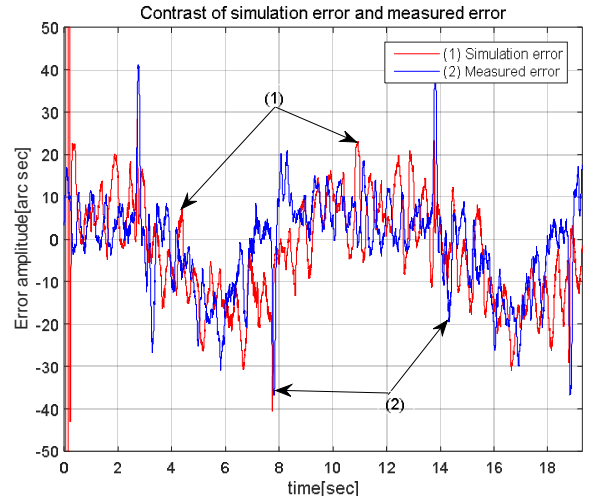

Fig. 11 Comparison of simulation error and measured error

Apparently see from the Fig. 11, the simulation result is highly matched with the measured error data. Therefore, we get a more accurate nonlinear model of the coarse tracking system.

Simulink complete simulation diagram shown in Fig. 12. Each module is marked in the diagram.

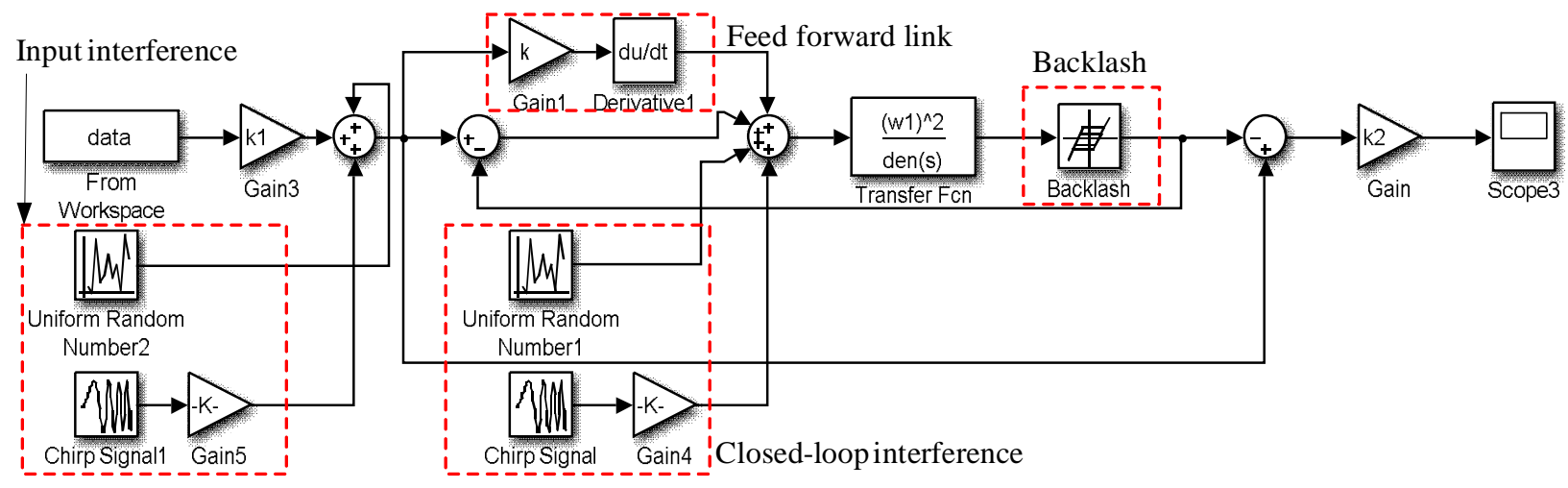

Fig. 12 Simulink complete simulation diagram

From the above process analysis inferred:

(1). Interference location is mainly in the closed-loop; 
(2). Interference causes jitter in the error, and the backlash results in a large jump.

Therefore, we used the simulation modeling method to obtain the model as the actual coarse tracking model, the closed-loop transfer function is fellow as:

$$
G(s)=\frac{57.8455^{2}(1+0.0242 s)}{s^{2}+2 \times 0.69 \times 57.8455 s+57.8455^{2}} .
$$

Backlash: $41 \times \pi / 360 \times 180=1.9877 \times 10^{-4}$.

Interference 1: non-unit random number, the maximum value of 10 seconds, the minimum -7 seconds, sampling time 0.2 seconds;

Interference 2: a sine wave with a frequency of $2 \mathrm{~Hz}$ and a gain of 0.00003 .

\section{Conclusions}

In this paper, we proposed a new modeling method, which combines analysis and simulation modeling to model the coarse tracking system in the composite axis system. As shown in Fig.11, the simulation error matched well with the measured error, and a more accurate nonlinear model with interference is obtained. In practice, the overall design of the system and the selection of the precision tracking device have been verified, and the method has engineering practicability.

\section{References}

[1] Shaojin Chen, Hongke Wang and Leigang Zheng: Fire Control \& Command Control.Vol. 37, No.4 (2012) (In Chinese).

[2] Ge Ren:Optoelectronic Engineering. (1995), p.41-46.

[3] Tingxia Liu: the research of Compund-axis Servo Control Technique of O-E Tracking System [D].Changchun: Chinese Academy of Science. 2005. (In Chinese)

[4] Ljung L, Hjalmarsson H, Ohlsson H: European Journal of Control. (2011).

[5] T. Soderstrom. Accuracy analysis of the frisch scheme for identifying errors-in-variables systems. IEEE (Transactions on Automatic Control). 2007.

[6] Ding F, Chen T: International Journal of Adaptive Control and Signal Processing. (2004).

[7] J. W. Fowler: Journal of Low Temperature Physics. Vol.176,(2014)p.414-420.

[8] Cover,T.M.,Thomas,J.A: Elements of Information Theory .(2006)

[9] Feng Ding: New Theory of System Identification[M]. Beijng: Science Press, (2013), pp 199-201 (In Chinese)

[10]Rubio, José De Jesús. SOFMLS: IEEE (Transactions on Fuzzy Systems),(2009).

[11]Leontaritis IJ, Billings SA: International Journal of Control,(1985).

[12] Songhe Zhou, Liexuan Xu: Engineering Mechanics.(2007). 